\title{
An Anthurium Growth Environment Monitoring System Based on Wireless Sensor Network
}

\author{
https://doi.org/10.3991/ijoe.v15i05.9382 \\ Jianqing Huang ${ }^{\bowtie)}$, Debing Liu, Qi Yuan \\ Hainan University, Danzhou, China \\ hjqoffice@ 163.com
}

\begin{abstract}
Anthurium is known as a famous and precious cut flower in the world, but its growth and ornamental effect are easily affected by environmental conditions such as temperature, humidity and light intensity. An environment parameter monitoring system based on wireless sensor network is proposed to enhance the flower production efficiency. The proposed system uses sensor nodes to acquire data of air temperature and humidity, light intensity and soil temperature and humidity, sink node to collect data from sensor nodes through wireless sensor network and send data to the PC of monitoring center. By using MSP430F149 as the main controller, nRF905 as the communication module, and AM2306, GY -30 and SMTS-II-485X as the air temperature and humidity, light intensity and soil temperature and humidity sensors, the hardware of the wireless sensor network node is realized. The node software is developed based on IAR Embedded Workbench and the computer monitoring software by VB6.0. The results show that the proposed system which is accurate and stable can make real-time monitoring of anthurium growth environment in a large scale. Therefore it can be widely applied in agricultural environmental monitoring.
\end{abstract}

Keywords-Wireless sensor network, anthurium growth environment, monitoring system, sensor nodes

\section{$1 \quad$ Introduction}

Anthurium, a perennial evergreen herbaceous plant from the tropical rain forest of South America, is known as a famous and precious cut flower in the world with its beautiful bright ornamental foliage, unique shape and long flowering period. But the quality properties of the anthurium are apt to be affected by environmental parameters such as air temperature and humidity, light intensity, and soil temperature and humidity, etc. For example, the suitable air temperature scopes of daytime and night are 25 degrees $\mathrm{C} \sim 28 \mathrm{C}$ and 19 degrees $\mathrm{C} \sim 21 \mathrm{C}$ for the growth of the Anthurium. When air temperature is below 15 degrees $\mathrm{C}$ or above 35 degrees $\mathrm{C}$ for a long time, anthurium will grow slowly, stop growing, and even die [1]. So it is necessary to establish a monitoring system to realize realtime and online auto monitoring growth 
environmental parameters of anthurium, so as to improve the anthurium production and efficiency.

In the past, manual monitoring method of flower growth environmental parameters was used, but it had some shortcomings, such as inefficiency, big man-made error and low-level automatization, so it could not meet the requirement in measurement of flower growth environmental parameters. So far some flower production companies have installed a wired monitoring system, realizing realtime and online auto monitoring flower growth environmental parameters. However, the difficulity of the installion of wired monitoring system is due to lay massive electric cable, so there are some disadvantages such as wiring difficulties, poor anti-interference ability and high cost. In recent years, wireless sensor network, as its advantages of no wiring, low cost and easy installation, has widely used in crop growth environmental monitoring aspect. But now there is a lack of research on monitoring anthurium growth environment by wireless sensor network technology.

In this paper, regarding the acquisition, processing, and communication of air temperature and humidity, light intensity, and soil temperature and humidity information are the research objects. By wireless sensor network technology, a kind of anthurium growth environment monitoring system is designed. The system uses high-precision air temperature and humidity sensor AM2306, light intensity sensor GY - 30, and soil temperature and humidity sensor SMTS-II-485X to measure the anthurium growth environmental information, which improves the accuracy of data acquisition and realizes multi-parameter measurement with a single node. The system constructs wireless network nodes with wireless communication module, no wiring, and easy operation, will enhance the flower production efficiency, thus has a good application prospect.

\section{State of the Art}

At present, flower growth environment monitoring mainly includes artificial timing measurement and real-time automatic measurement methods. Artificial timing measurement method is that flower managers measure flower growth environmental factors every day in a certain time through temperature meter, humidity meter, test paper and so on. This uses a lot of manpower, cannot achieve large-scale measurement, and cannot be real-time monitoring change in flower growth environment. As a result, flower managers cannot find abnormalities to respond in time and ensure that flower grows healthily and safely [2]. Real-time automatic measurement method is that flower growth environment factors are measured automatically and continuously through a system composed of instruments, computers and other communication equipments. This can collect large amounts of data quickly, real-time monitor changes in flower growth environment, but many of the existing monitoring systems are in need of wiring massive electric cable with some disadvantages such as wiring difficulties, poor anti-interference ability, high cost and difficult construction technology [3]. 
In recent years, wireless sensor network has become a hotspot of research field as its unique value and advantages in many fields. Wireless sensor network consists of a large number of small wireless sensor nodes, which form an Ad Hoc style network, sense and process various types of monitoring object data information, and send the data information to users [4]. Compared with the traditional networks, wireless sensor networks have the advantages of low power, low cost, no wiring and easy installation, thus has been widely applied in agricultural production fields [5]. For example, Mancuso Marco used Sensicast Company's RTD204 module to design a system based on wireless sensor network for monitoring environmental variables such as air temperature, air humidity and soil temperature in a tomato greenhouse [6]. Park DH designed a greenhouse environment monitoring system based on ZigBee, which can monitor air temperature and humidity, and leaf temperature and humidity in real time, and can also control greenhouse temperature and humidity automatically [7]. Chenguan Sun designed an intelligent greenhouse environmental monitoring system by using wireless sensor network technology, VB programme and flash technique [8]. Feng Gao and others designed a real-time and remote monitoring system for crop water status based on wireless sensor network, which can monitor crop water deficit and some physiological indices [9]. In conclusion, wireless sensor network is very suitable for agricultural production fields.

\section{Methods}

\subsection{System overall structure design}

The system is composed of four parts: sensor nodes, router nodes, a sink node and a PC. Sensor nodes, router nodes and a sink node consist of the wireless sensor network of the system. Sensor nodes are deployed randomly in the greenhouse monitor area, and router nodes are distributed around sensor nodes, and flower Management office is provided with a sink connected with a computer through RS232 serial port to form a separate network. The system structure diagram is shown in figure 1 .

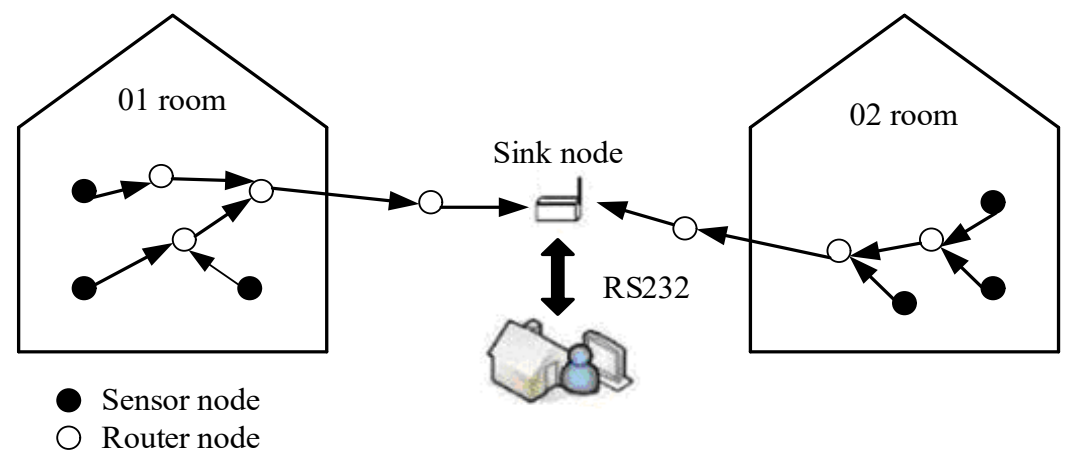

Fig. 1. System structure diagram 


\subsection{Hardware design of monitoring system}

Hardware design of sensor node: The main function of the sensor node is to acquire and process the air temperature and humidity, light intensity, soil temperature and humidity data of anthurium growth environment, and to wirelessly send data to router nodes or receive data from router nodes. In terms of hardware architecture, the sensor node is mainly composed of sensor module, processor module, wireless communication module and power supply module. The sensor module composed of air temperature and humidity sensor, light intensity sensor, and soil temperature and humidity sensor and their interface circuits, is responsible for collecting environmental data. The processor module composed of the processor chip MCU and its peripheral circuits, is responsible for task scheduling, data processing, communication protocol and so on. The wireless communication module composed of radio frequency chip and its peripheral circuits, is responsible for wirelessly sending data to router nodes and receiving data from router nodes. The power supply module provides energy for each module's normal operation. The picture of the sensor node is shown in figure 2.

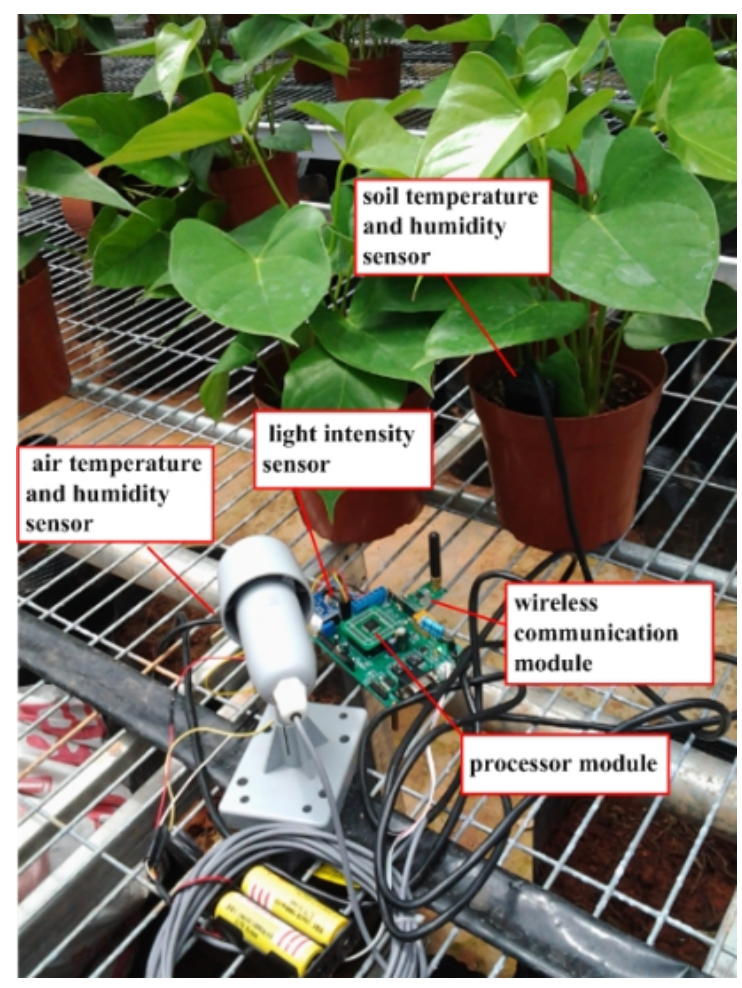

Fig. 2. The picture of sensor node

The sensor module: In order to meet the requirements of anthurium growth environment monitoring, the sensor module uses high-precision sensors such as AM2306, 
GY -30 and SMTS-II-485X to measure air temperature and humidity, light intensity and soil temperature and humidity information.

AM2306 sensor, launched by China Guangzhou Lexiang Company, is used to measure air temperature and humidity. It includes one capacitive humidity sensor and one high-precision temperature sensor connected with 8-bit single chip microcomputer. And its advantages mainly reflect in quick response, anti-interference and high cost performance. Its temperature measurement precision is \pm 1 degrees $\mathrm{C}$ and humidity measurement precision is $\pm 2 \% \mathrm{RH}$. AM2306 has a unique single bus interface, the DATA pin of which is connected with the P2.3 pin of MSP430F149 and the power through $5.1 \mathrm{k} \Omega$ resistor to realize bidirectional communication with $\mathrm{MCU}$. The interface circuit between AM2306 and MSP430F149 is shown in figure 3.

GY - 30 sensor with BH1750FVI chip, launched by Japan ROHM Semiconductor Company, is a light intensity sensor with excellent spectral sensitivity. Its light intensity measurement range is $0 \sim 655351 \mathrm{x}$, and it has a unique I2C serial bus interface, the SCL pin of which is connected with the P5.6 pin of MSP430F149 to receive clock signal from MCU, the SDA pin of which is connected with the P5.7 pin of MSP430F149 to receive control information from MCU or send data to MCU. The interface circuit between GY-30 and MSP430F149 is shown in figure 4.

SMTS-II-485X sensor, launched by China Dalian Qi Feng science and Technology Company, is used to measure soil temperature and humidity for the surface and deep soil. Its temperature measurement precision is \pm 0.5 degrees $\mathrm{C}$, and humidity measurement precision is $\pm 3 \% \mathrm{RH}$ when humidity is below $53 \%$, otherwise it is $\pm 3 \% \mathrm{RH}$. SMTS-II-485X sensor has a unique RS485 industrial common interface, which communicates with MCU through SP485 chip. The RO pin of SP485, an output port used to send data to MCU, is connected with the P3.7 pin of MSP430F149. The RI pin of SP485, an input port used to receive data from MCU, is connected with the P3.6 pin of MSP430F149. The RE and DE pins of SP485, control ports used to set SP485 work mode, are connected with the P3.3 pin of MSP430F149. In the meantime the A and B pins of SP485, input/output ports used to send control information to SMTS-II-485X sensor or receive data of SMTS-II-485X sensor. The interface circuit between SMTSII-485X and MSP430F149 is shown in figure 5.

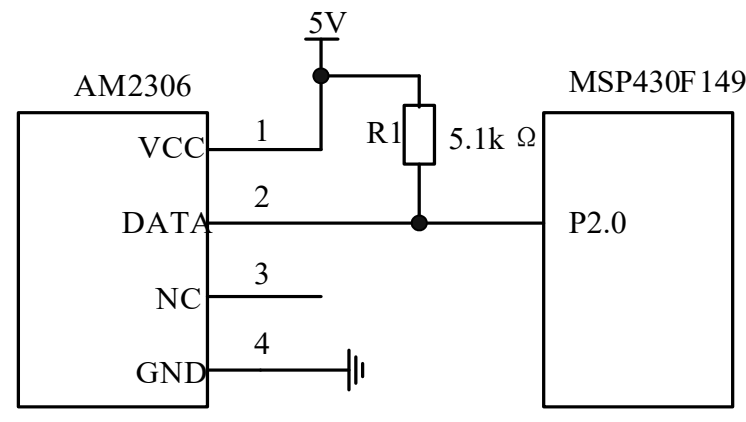

Fig. 3. Interface circuit between AM2306 and MSP430F149 


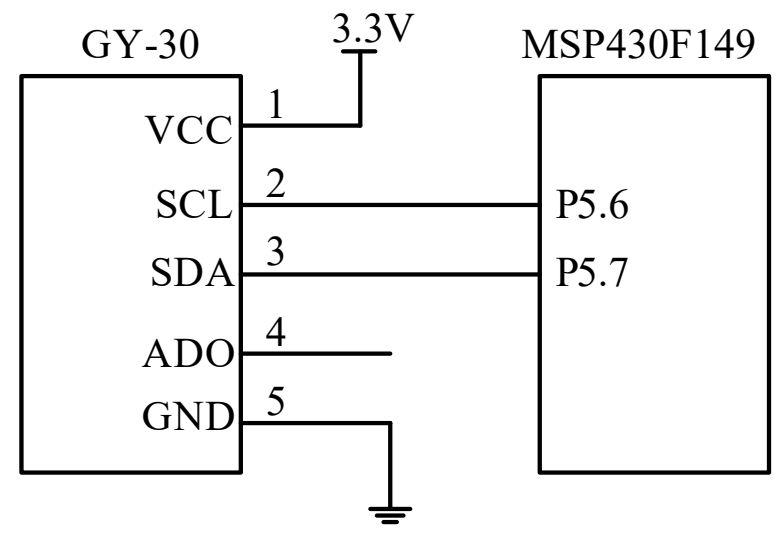

Fig. 4. Interface circuit between GY-30 and MSP430F149

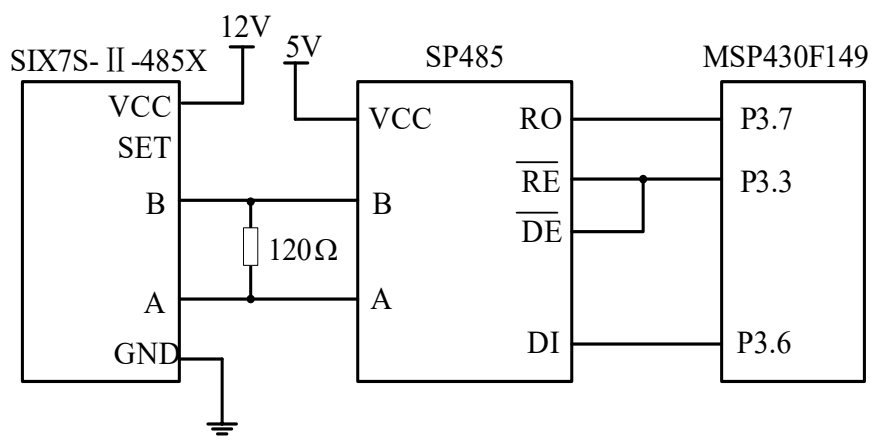

Fig. 5. Interface circuit between SMTS-II-485X and MSP430F149

The processor module: As the core part of sensor node, the processor module is mainly composed of MSP430F149 MCU and its peripheral circuits. MSP430F149 only needs $1.8 \mathrm{~V}-3.6 \mathrm{~V}$ voltage power supply, and its electric current is only $280 \mu \mathrm{A}$ at full speed running state, and $0.1 \mathrm{u}$ A at dormant state[10]. MSP430F149 is especially suitable for low power demand occasions with its low voltage and low power consumption.

The wireless communication module: The wireless communication module mainly composed of nRF905chip and its peripheral circuits, is used to wirelessly transmit or receive data. The nRF905chip, which is the RF $433 / 868 / 915 \mathrm{MHz}$ chip produced by NORDIC company [11], has three sets of interface, SPI transmission interface, state output interface and mode control interface. The SPI transmission interface is used to transform and exchange data with MCU through four pins, CSN, SCK, MISO and MOSI. These pins are connected with the P1.0, P1.2, P1.6 and P1.4 of MSP430F149 respectively. The state output interface is used to monitor nRF905 work state through three pins, $\mathrm{CD}, \mathrm{AM}$ and DR. These pins are connected with the P2.3, P2.2 and P2.1 of MSP430F149 respectively. The mode control interface is used 
to set working mode of nRF905 through three pins, TRX_CE, TX_EN, PWR_UP. These pins are connected with the P3.0, P3.2 and P2.5 of MSP430F149 respectively. The interface circuit between nRF905and MSP430F149 is shown in figure 6 .

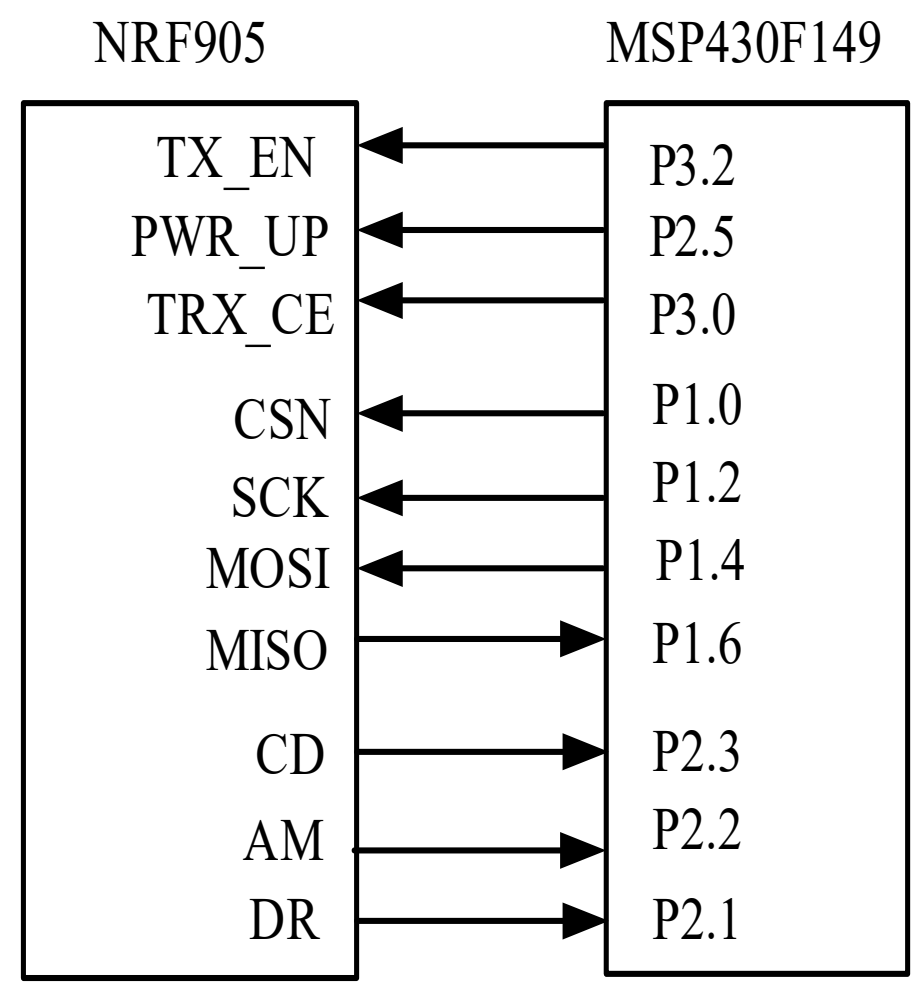

Fig. 6. Interface circuit between nRF905 and MSP430F149

The power supply module: The power supply module is the energy supply part of sensor node. Considering the requirements for outside monitoring, the power supply module uses $1200 \mathrm{mAh}$ and $3.2 \mathrm{~V}$ lithium-ion battery to provide electricity. The voltage of two Lithium batteries in series is transformed into 5V through LT1129-5, and then the voltage of $5 \mathrm{~V}$ is transformed into $3.3 \mathrm{~V}$ and $12 \mathrm{~V}$ through LT1129-3.3 and LT1930 respectively. $5 \mathrm{~V}$ is used to provide voltage for the air temperature and humidity sensor AM2306 and light intensity sensor $\mathrm{GY}-30$. And $3.3 \mathrm{~V}$ is used to provide voltage for the MSP430F149 MCU and nRF905 RF chip. Besides $12 \mathrm{~V}$ is used to provide voltage for the soil temperature and humidity sensor SMTS-II-485X. The power supply module structure is shown in figure 7 . 


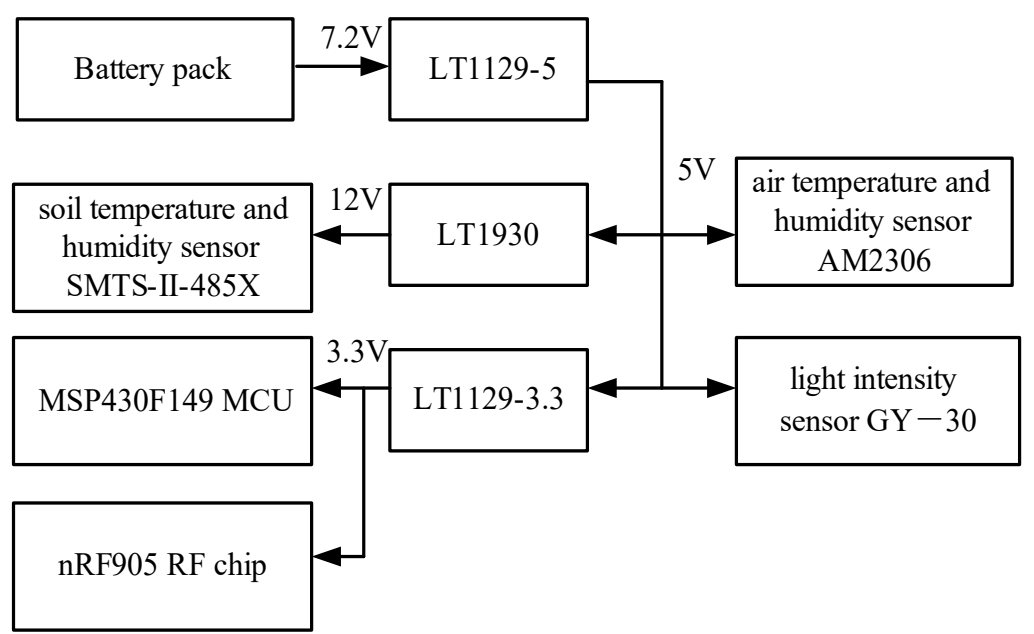

Fig. 7. The power supply module structure

Hardware design of router node: The main function of the router node is to wirelessly send data from sensor nodes to foreword nodes. In terms of hardware architecture, the router node is mainly composed of processor module, wireless communication module and power supply module. The router node only reduces the sensor module, and the other modules have the same hardware design as sensor node. The picture of the router node is shown in figure 8 .

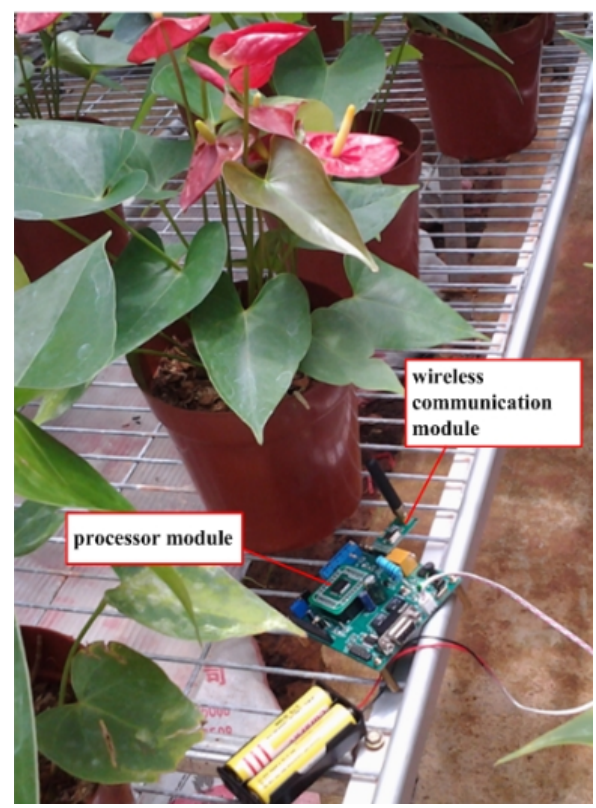

Fig. 8. The picture of router node 
Hardware design of sink node: The main function of the sink node is to collect all sensor node data by means of wireless communication, send the data to the PC of monitoring center, as well as send control signal to all nodes in the network for coordinating their work. In terms of hardware architecture, the sink node only increases serial communication module, and the other modules have the same hardware design as sensor node. The picture of the sink node is shown in figure 9 .

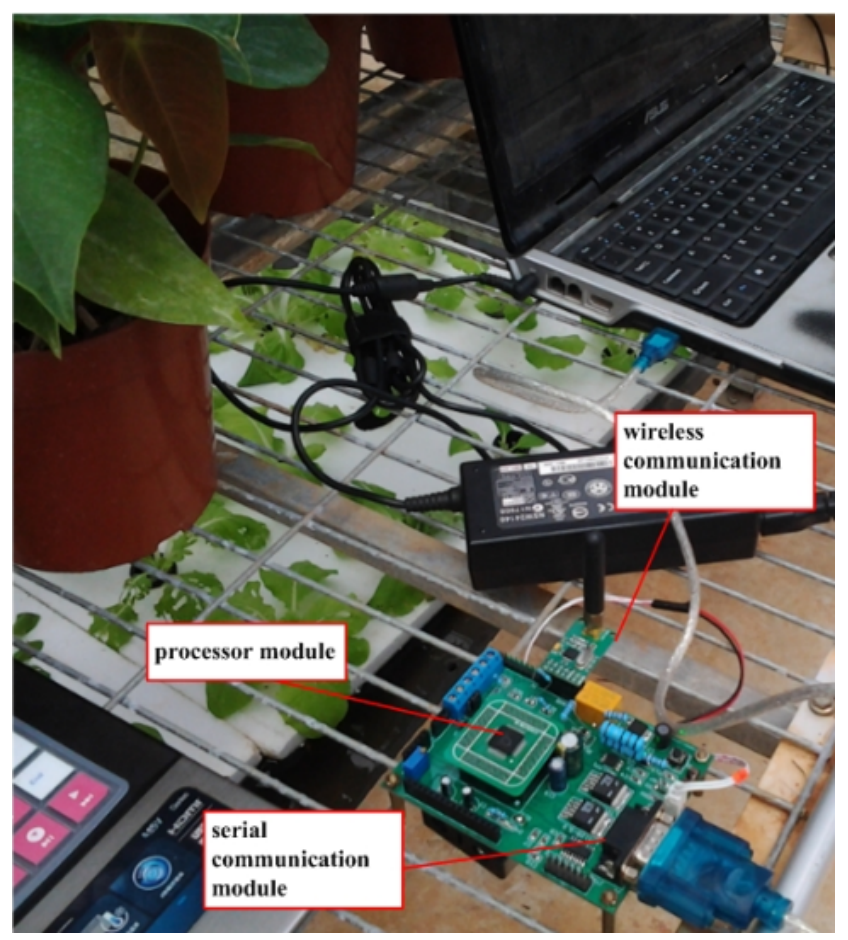

Fig. 9. The picture of sink node

\subsection{Software design of monitoring system}

According to the specific functions of the anthurium growth environment monitoring system, the software design includes the wireless network node software and computer monitoring software. As the core of the system software, the wireless network node software, which is composed of sensor node software, router node software and sink node software, is responsible for hardware initialization, data acquisition and processing, wireless transmission and other functions.Computer monitoring software is used to provide a visual human-computer interaction interface for flower producers.

Sensor node software design: The sensor node software, which is developed based on IAR Embedded Workbench, mainly includes hardware initialization, data acquisition and wireless communication. First of all, the sensor node initializes the 
hardware, including system clock, I/O port, timer and nRF905. Secondly, the node waits for the synchronize information sent by the sink node, and adjusts its own timer according to the synchronize information. And then the node enters the state of periodic data acquisition when a timer, which is used to mark the time of sending the data, starts running. The node can receive data from other nodes or send data to them till the time of sending the acquisition data is up. At this time, if the node's own data acquisition is archieved, it wirelessly sends the data to other nodes. Finally, the node enters into waiting for the synchronize information again. The sensor node software flow chart is shown in figure 10 .

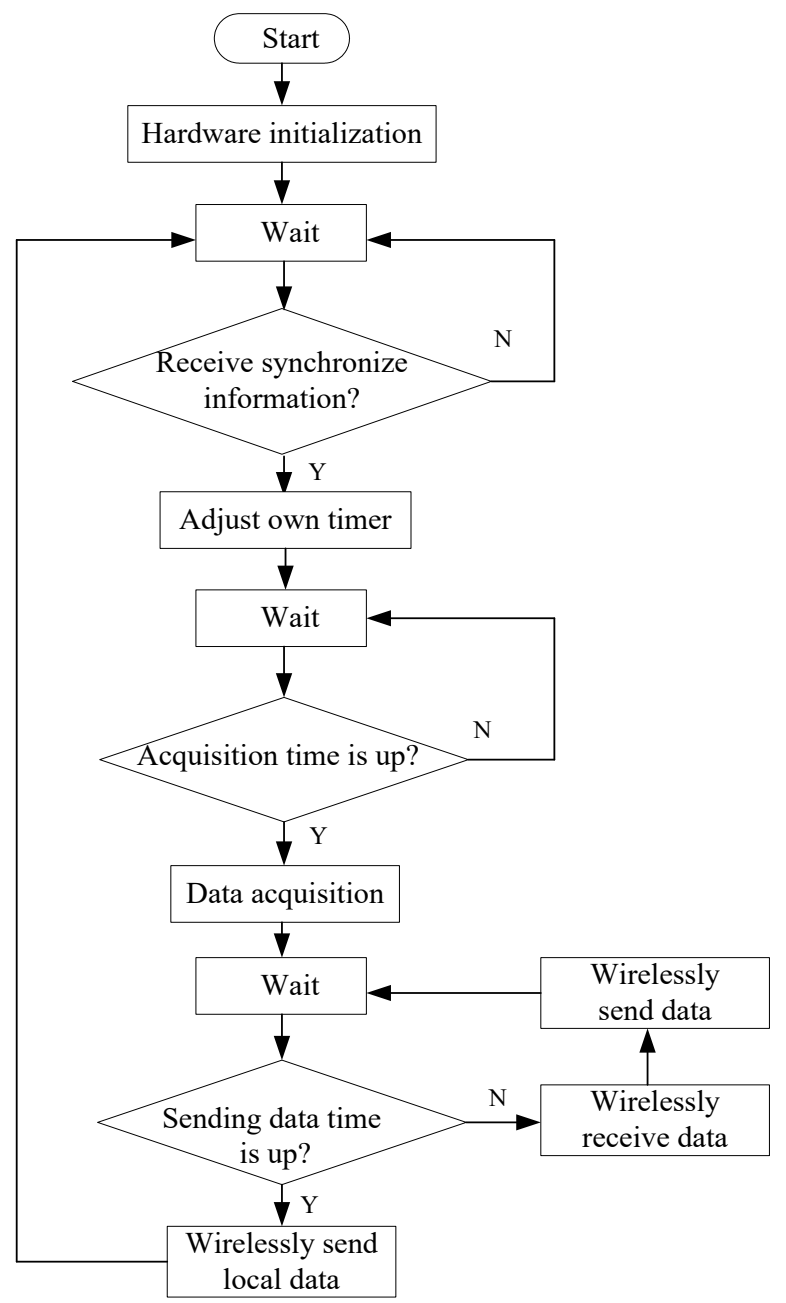

Fig. 10.The sensor node software flow chart

The synchronize information includes the node's timing rounds and the difference between node's comparator value and timer value, is sended by the sink node to coor- 
dinate network node's operation. Suppose that the sink node's and the sensor node's timing rounds are $\mathrm{R} 0$ and $\mathrm{R} 1$, and that their timer values are $\mathrm{T} 0$ and $\mathrm{T} 1$, and their comparator values are $\mathrm{C} 0$ and $\mathrm{C} 1$, thereby the sink node's and sensor node's differences between their comparator values and timer values are $\mathrm{C} 0-\mathrm{T} 0$ and $\mathrm{C} 1-\mathrm{T} 1$. After the network starts running, the sink node packs its own $\mathrm{R} 0$ value and $\mathrm{C} 0$ - $\mathrm{T} 0$ value into a synchronize information package, and wirelessly sends it to all nodes of the network. After receiving the package, the sensor node parses it and reads out the R0 value and $\mathrm{C} 0-\mathrm{T} 0$ value, and then changes its own timing round $\mathrm{R} 1$ and timer value $\mathrm{T} 1$ as shown in the following formula:

$$
\begin{gathered}
R_{1}=R_{0} \\
T_{1}=C_{1}-\left(C_{0}-T_{0}\right)
\end{gathered}
$$

By this way the sink node can coordinate all network nodes' work state, thus greatly reducing data loss of wireless sensor network.

The time interval of data acquisition is set to 30 minutes, which is realized by the timer Timer_A and comparator CCR0 of MSP430F149. The auxiliary clock ACLK of MSP430F149 with a frequency of $32 \mathrm{kHz}$ is selected as the reference clock of the Timer_A after the eighth sub-frequency, and the values of Timer_A and comparator CCR 0 are set to 0 and 61451. After the Timer_A starts runing, its value is continuously added till equal to the value of comparator CCR 0 when a timer interrupt is raised, at this time the timing round $\mathrm{R} 0$ is increased by one. If the timing round R0 is added to 120 , the data acquisition is end. The time interval of Timer_A is 15 seconds, while the time interval of data acquisition is 30 minutes, as shown in the following formula:

$$
\begin{gathered}
\mathrm{t}_{1}=\mathrm{CCR} 0 \times \frac{1}{\mathrm{f}_{\mathrm{ACK}} / 8}=61451 \times \frac{1}{\left(32 \times 10^{3}\right) / 8} \approx 15 \mathrm{~s} \\
\mathrm{t}_{2}=\mathrm{R}_{0} \times \mathrm{t}_{1}=120 \times 15=1800 \mathrm{~s}=30 \mathrm{~min}
\end{gathered}
$$

After completion of the data acquisition, the sensor node is in a state of receiving/transferring data during the timer of sending the acquisition data runs. First, the nRF905's PWR_UP and TRX_CE pins are set to high electric level, and TX_EN pin low electric level to make the sensor node in a state of receiving data, besides the external interrupt is allowed through the P2.1 pin of MSP430F149 connected with the DR pin of nRF905. Once receiving data from other nodes when the DR pin of nRF905 becomes high electric level, the node enters an external interrupt program where the data is readed out through the CSN, SCK and MISO pins of nRF905, and saves. After this, the nRF905's PWR_UP, TRX_CE and TX_EN pins are all set to high electric level to make the sensor node in a state of sending data, thereby the receiving data can be wirelessly sended again. If the time of sending the acquisition data is up, the node wirelessly send its own acquisition data.

Router node software design: In the wireless network, the main function of router node is to wirelessly send data acquired by sensor nodes to other nodes. The work 
task of router node is roughly the same as sensor node but without data acquisition, thus its software has not data acquisition module, others is the same as sensor node.

Sink node software design: After collecting all sensor node acquisition, the sink node sends them to the PC of monitoring center. In addition, it also sends synchronize information to all nodes in the network. The sink node software, which is developed based on IAR Embedded Workbench, mainly includes hardware initialization, wireless communication, and serial communication. First of all, the sink node initializes the hardware, including system clock, I/O port, timer, nRF905 and serial port. Secondly, the sink node wirelessly sends synchronize information, and then goes into the state of periodic sleep. It does not be waked up to go into the working state until the sleep time is end. After be waked up, it opens the wireless receiver module to receive data and send them to PC of monitoring center trough serial interface. Finally, the sink node wirelessly sends the synchronize information to all nodes, and a new cycle will begin again .The sink node software flow chart is shown in figure 11 .

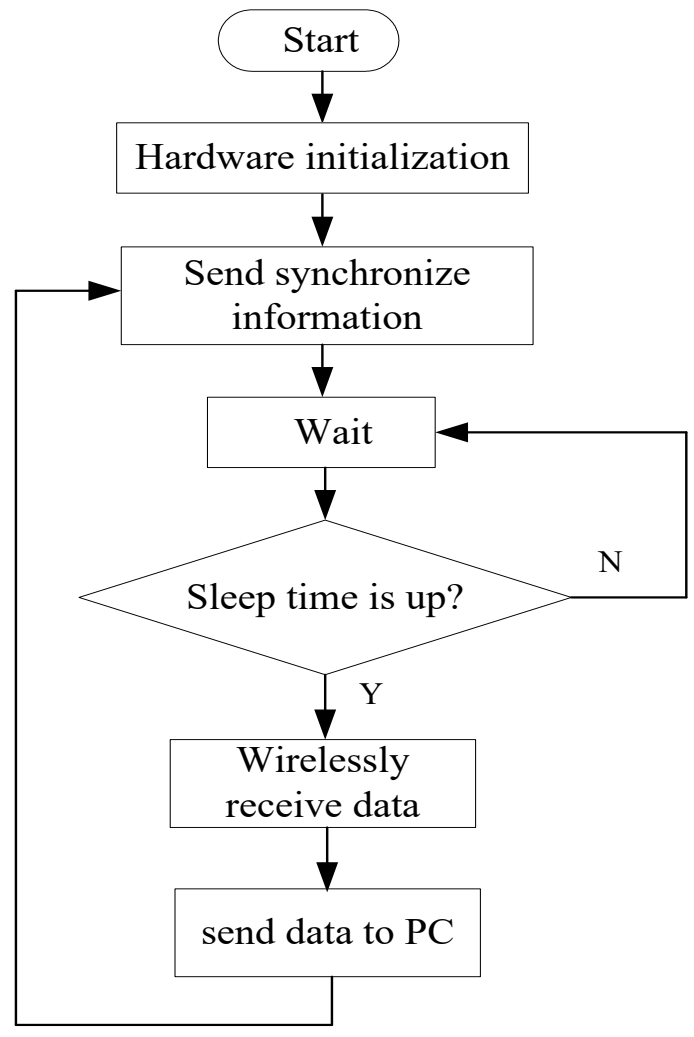

Fig. 11. The sink node software flow chart

Computer monitoring software design: The computer monitoring software makes it possible for flower managers to look up real-time data. The software, developed by VB6.0, communicates with the sink node through a serial communication 
component MSComm. If the sink node sends data to the serial interface of PC, an OnComm event of MSComm control is raised, in which the $\mathrm{PC}$ receives data and save data in the form of excel file by calling a serial interrupt program. The software has some functions, such as real-time data storage, real-time curve showing, report query, parameters setting, and over-limit alarming. The computer monitoring software interface is shown in figure 12.

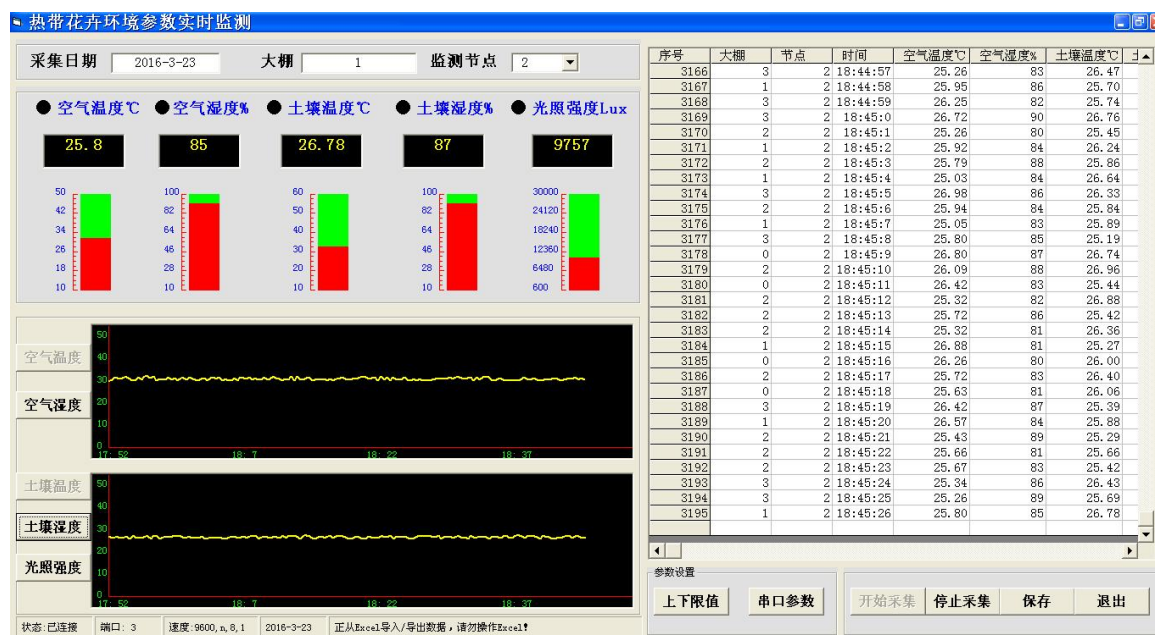

Fig. 12. The computer monitoring software interface

\section{System Test}

\subsection{System function test}

The system consists of four sensor nodes, one sink node and three router nodes. The sensor nodes were deployed in different motoring areas of an anthurium greenhouse of Hainan University, and the router nodes were deployed in the corridor of the motoring area, as well as the sink connected with PC, was deployed in the office of monitoring center. These nodes formed a self-organized and multi-hop wireless sensor network. The sensor node collected data once a minute, which was transmitted to the monitoring computer software as shown in figure 12. From figure 12, it is clearly shown that real-time air temperature and humidity, light intensity, and soil temperature and humidity data are displayed through curve and table. When the real time monitoring data of certain environmental parameter exceeds the limit preset, the small black circle next to this parameter will be blinking on the display to remind user.

\subsection{Data acquisition test}

In order to validate the proposed system, the measurement data collected by the sensor node was compared with standard measurement instruments, including air 
temperature and humidity meter HTC-1, light intensity meter TM830M, and soil temperature and humidity meter QSY-QS-WT. HTC-1 temperature measurement accuracy is $\pm 1{ }^{\circ} \mathrm{C}$, and its humidity measurement accuracy is $\pm 5 \% \mathrm{RH}$. TM830M light intensity measurement accuracy is $\pm 3 \% \mathrm{rdg} \pm 0.5 \%$ f.s. QSY-QS-WT temperature measurement accuracy is $\pm 0.3{ }^{\circ} \mathrm{C}$, and its humidity measurement accuracy is $\pm 3 \%$ RH.

Compared with the standard measurement instruments, the sensor node measurement curves generally share similar trends, and its measurement data are close to standard instrument, as shown in Fig. 13 to 17 . It is shown that the environmental parameter data collected by the sensor node of the proposed system is reliable.

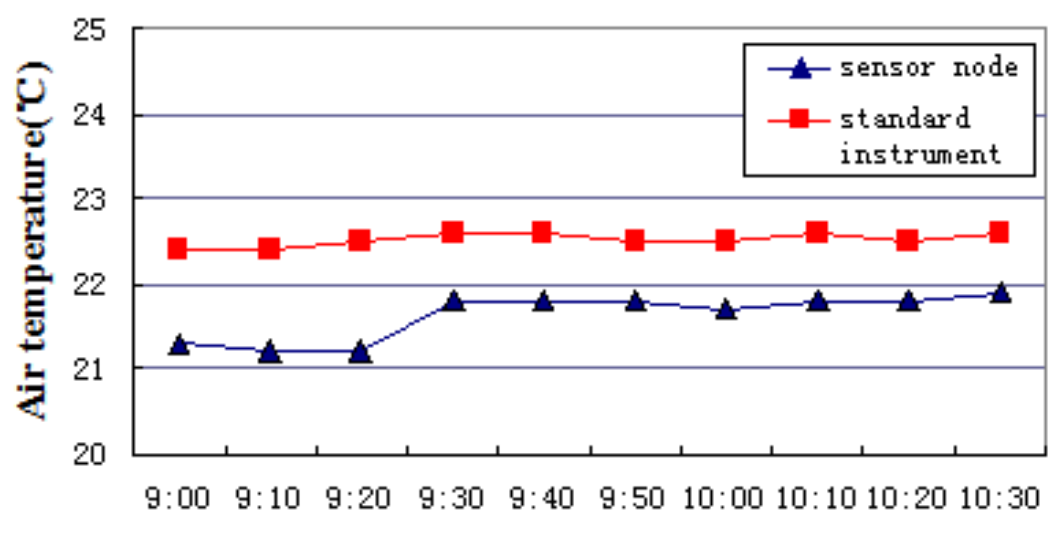

Time

Fig. 13. Air temperature measurement

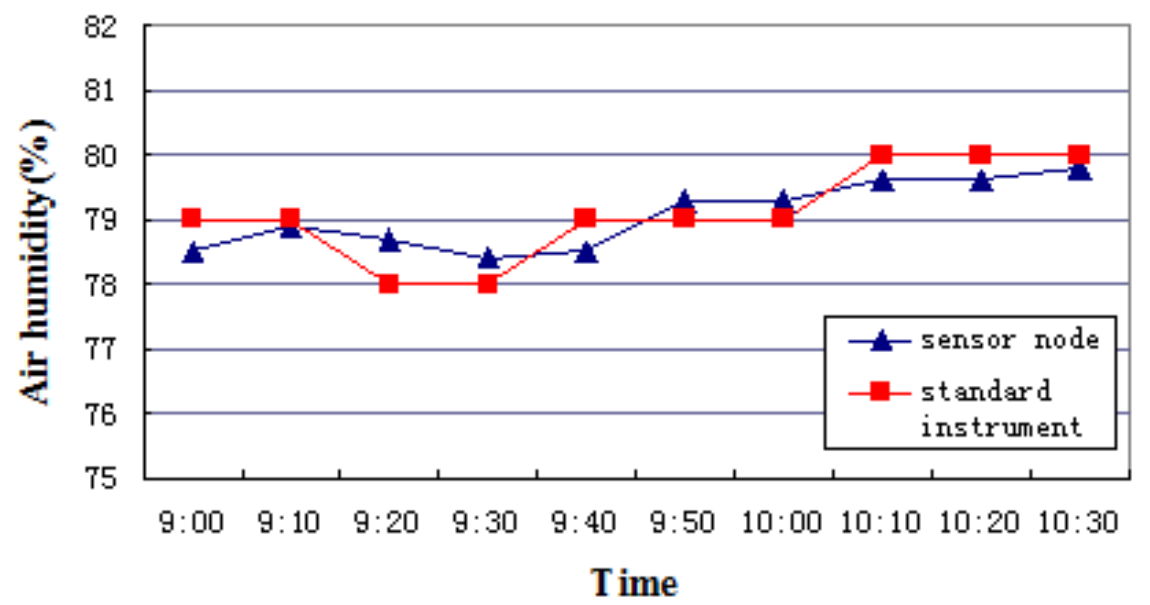

Fig. 14.Air humidity measurement 


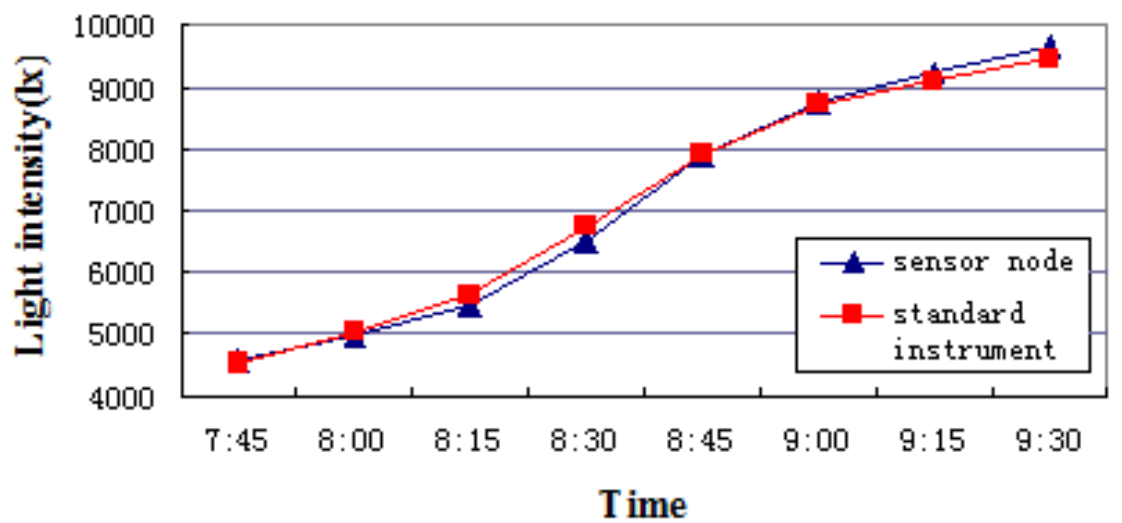

Fig. 15. Light intensity measurement

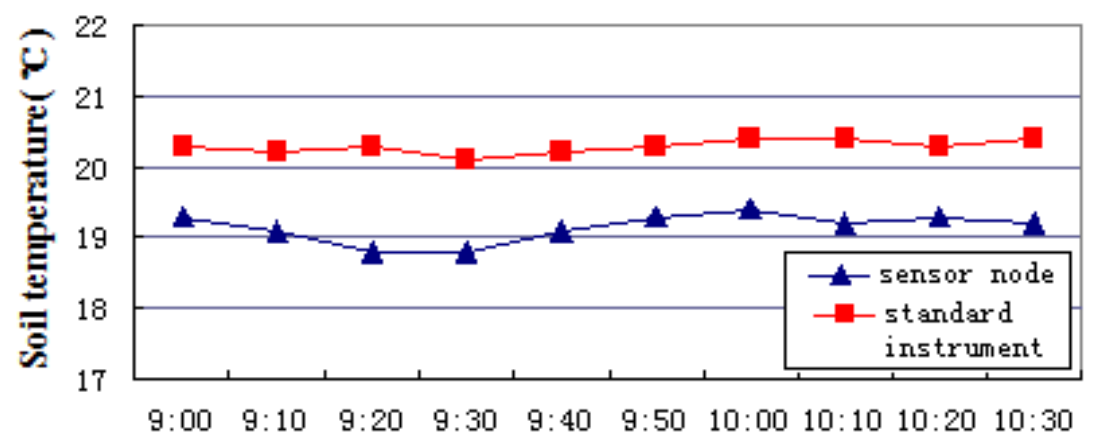

T ime

Fig. 16. Soil temperature measurement

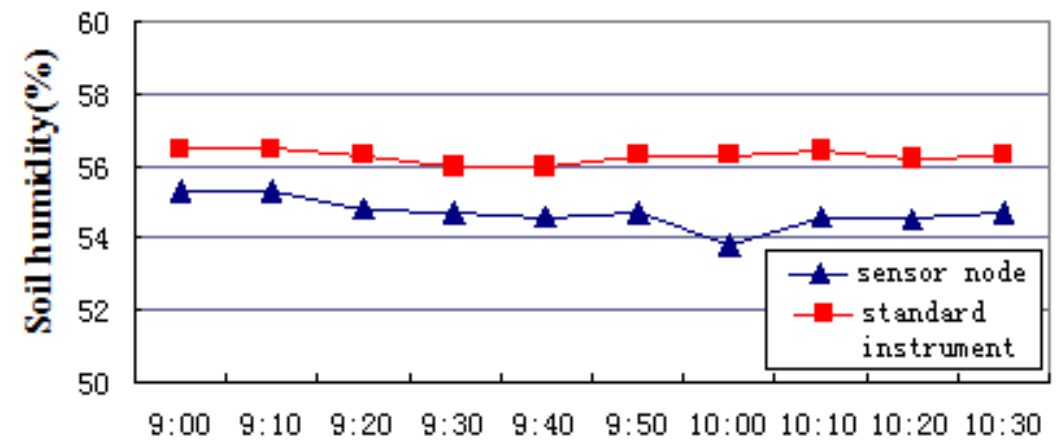

Time

Fig. 17. Soil humidity measurement 


\section{Conclusion}

In this paper, a kind of monitoring system based on wireless sensor network for anthurium growth environment is introduced and the design of hardware and software is discussed in detail. In addition, the feasibility and practicability of the system are verified by test. The experimental results show that it is very suitable for the system to be applied to agricultural environmental monitoring for its low cost, good stability, good flexibility, and higher accuracy.

\section{Acknowledgement}

This work has been financially supported by Natural Science Fund of Hainan province (20153096). The authors would like to thank the teachers of Institute of Tropical Agriculture and Forestry of Hainan University for the assistance on the system test.

\section{$7 \quad$ References}

[1] Liu, Z., Chen, Y., Zhou, X. (2012). The disease and pest control of anthurium. China Flowers \& Horticulture, 12, 34-37.

[2] Chang, K., Lee, J., Jun, C. H., \& Chung, H. (2016). Acquisition of a series of temperature varied sample spectra to induce characteristic structural changes of components and selection of target-descriptive variables among them for multivariate analysis to improve accuracy. Applied Spectroscopy Reviews, 51(7-9), 718-734. https://doi.org/10.10 80/05704928.2016.1167069.

[3] Gan, B., Jin, S. (2018). Design of early warning system based on wireless sensor network. International Journal of Online Engineering, 14(1), 66-76. https://doi.org/10.3 991/ijoe.v14i01.8060.

[4] Klemenjak, C., Egarter, D., \& Elmenreich, W. (2016). Yomo: the arduino-based smart metering board. Computer Science - Research and Development, 31(1-2), 97-103.

[5] http://dx.doi.org/10.1007/s00450-014-0290-8.

[6] Sun, Y., Shen, M., Lu, M., Lin, X., \& Liu, L. (2011). Research status and prospect of wireless sensor network in agriculture. Acta Agriculturae Zhejiangensis, 23(3):639-644.

[7] Marco, M., Franco, B. (2006). A wireless sensors network for monitoring environmental variable in a tomato greenhouse. IEEE International Workshop on Factory Communication Systems, Proceedings, 1,107 -110. https://doi.org/10.1109/WFCS.2006.1704135.

[8] Park, D. H., Kang, B. J., Cho, K. R., Shin, C. S., ... \& Yang, W., M. (2009). A study on greenhouse automatic control system based on wireless sensor network. Wireless Personal Communications, 56(1), 117 -130. https://doi.org/10.1007/s11277-009-9881-2.

[9] Sun C. (2007). Design of intelligent greenhouse system based on wireless sensor network. Journal of tianjin university of technology and education, 17(4), 19-22.

[10] Gao, F., Yu, L., Zhang, W., Xu, Q., \& Yu, L. (2009). Research and design of crop water status monitoring system based on wireless sensor networks. Transactions of the CSAE, 25(2), 107-112.

[11] Wang, W., Luo, X., Sun, D. (2010). Design of wireless sensor network node for data transmission in tea plantations. Transaction of the CSAE, 27, 169-173. 
[12] Hu, P., Liu, H. (2017). Design and Application of Electrical Equipment Overheating Monitoring Based on Wireless Sensor. International Journal of Online Engineering,13(7),25-35. https://doi.org/10.3991/ijoe.v13i07.7277.

\section{Authors}

Jianqing Huang is with Institute of Tropical Agriculture and Forestry Technology of Hainan University, Danzhou, China.

Qi Yuan is with College of Mechanical \& Electrical Engineering of Hainan University, Haikou, China.

Debing Liu is with Tropical Agriculture and Forestry Technology of Hainan University of Institute, Danzhou, China.

Article submitted 2018-08-12. Resubmitted 2018-10-08. Final acceptance 2018-10-26. Final version published as submitted by the authors. 